\title{
An Energy Efficient Data Transfer Mechanism for Wireless Sensor Network
}

\author{
Jyoti Saraswat $^{1}$ and Partha Pratim Bhattacharya ${ }^{2}$ \\ Department of Electronics and Communication Engineering \\ Faculty of Engineering and Technology \\ Mody Institute of Technology \& Science (Deemed University) \\ Lakshmangarh, Rajasthan - 332311, India. \\ 1jyotisaraswat.mit@gmail.com \\ 르ereispartha@gmail.com
}

\begin{abstract}
Wireless Sensor Networks (WSNs) are generally energy and resource constrained. In most WSN applications the traffic pattern is from sensor-to-sink and for effective utilization of available resources in network data aggregation is employed. If a data packet is lost due to node failure or collision the correlated information content by data packets is lost. Existing protocols that provide reliable data transfer for sensor-to-sink traffic are either not energy efficient or they provide reliability at the event level. Energy efficiency can be improved by employing proper duty cycle values. By extending the concept of monitors the proposed protocol provides packet level reliability and improves the energy efficiency by employing duty cycles. To further decrease the energy consumption only a subset of nodes is chosen as active nodes to transfer the data. The performance of the proposed protocol is evaluated using Matlab. Results show that protocol has significant improvement in terms of energy saving, throughput and packet delivery ratio.
\end{abstract}

\section{General Terms}

Wireless Sensor Network

\section{Indexing terms}

Wireless sensor network, duty cycle, energy efficiency, energy consumption, throughput, protocol, packet delivery ratio

\section{Council for Innovative Research}

Peer Review Research Publishing System

Journal: INTERNATIONAL JOURNAL OF COMPUTERS \& TECHNOLOGY

Vol 6, No 3

editor@cirworld.com

www.cirworld.com, member.cirworld.com 


\section{INTRODUCTION}

Wireless sensor network (WSN) [1] consists of a large amount of small sensor nodes. These small sensors have the ability of data processing, sensing and communicating with each other and to the outside world through the external base station. The Base Station in turn queries the sensor nodes for information and is responsible for collecting the data and relaying it to other networks. The primary traffic pattern in most WSN applications is sensor-to-sink, although the sink occasionally sends control packets to the sensor nodes. These types of traffic patterns from sensor nodes to Base Station limit the network scalability as the nodes closer to the Base Station come across heavy traffic and consume their energy rapidly.

A sensor-to-sink data transport technique that provides packet-level reliability and supporting in-network data aggregation was proposed in which the inactive sensors were dynamically started working as monitors. By utilizing the information provided by monitors, more reliable loss detection can be achieved in case of sudden node failures. However, this technique is not energy efficient due to the idle listening behavior of the sensor nodes, which deplete energy frequently. Sensor nodes spend a considerable amount of time in monitoring the environment while only some spend a small portion of time to report sporadic events. The energy consumption in the idle listening state is very high compared to the transmission or receiving state. By employing duty cycle energy efficiency can be improved.

In [2], active and inactive nodes are chosen and from the subset of inactive nodes, subset of nodes called monitors is chosen. Due to this energy consumption of the nodes can be reduced. Duty cycles are employed for all the active nodes to further reduce the energy consumption.

The rest of the paper is organized as follows: we briefly described related works in Section 2. The proposed model is presented in Section 3. Results and discussion are reported in Section 4 followed by conclusion in Section 5.

\section{RELATED WORKS}

In wireless sensor network research, the energy efficiency and the reliable data transfer play a very important role. Many protocols have been proposed to provide energy efficiency and reliable data transfer of packets in WSNs.

Honghai Zhang et. al [3] designed an algorithm based on the derived upper bound, an algorithm that sub optimally schedules node activities to maximize the $\alpha$-lifetime of a sensor network where the time is normalized to be the lifetime of each sensor node. In [3], the node locations and two upper bounds of the $\alpha$-lifetime are allocated. Based on the derived upper bound, an algorithm that sub optimally schedules node activities to maximize the $\alpha$-lifetime of a sensor network is designed. Simulation results show that the proposed algorithm achieves around $90 \%$ of the derived upper bound. This implies that the derived upper bounds are rather tight and the proposed algorithm is close to optimal.

MS Pawar et. al [4] discussed the effect on lifetime, and energy consumption during transmission, listen (with different data packet size), idle and sleep states. The energy consumption of WSN node is measured in different operational states, e.g., idle, listen, transmit and sleep. These results are used to predict the WSN node life time with variable duty cycle for sleep time. They concluded that sleep current is an important parameter to predict the life time of WSN node. Almost $79.84 \%$ to $83.86 \%$ of total energy is consumed in sleep state. Reduction of WSN node sleep state current $\mathrm{I}_{\text {sle ep }}$ from $64 \mu \mathrm{A}$ to $9 \mu \mathrm{A}$ has shown improvement in lifetime by 193 days for the $3.3 \mathrm{~V}, 130 \mathrm{mAh}$ battery. It is also analyzed that the WSN node lifetime also depends on the packet size of data. Data packet size is inversely proportional to the life time of the node. As data packet size is increased, the lifetime of the battery is decreased.

Yuqun Zhang et. al [5] proposed an adaptation method for the derived distance-based duty cycle based on local observed traffic. In this paper, the Packet Delivery Ratio (PDR) values are achieved by three methods. According to their simulation, in all the three methods the PDR results are very close and higher than $97 \%$ for light traffic loads. With an increase in traffic load, the constant duty cycle method performs the best because its higher duty cycle can provide more awake nodes to participate in data routing. The slightly worse performance of TDDCA (Traffic- Adaptive Distance-based Duty Cycle Assignment) compared to the constant duty cycle method indicates that the fixed increments and decrements in duty cycle is not efficient in terms of PDR. TDDCA and DDCA (Distance-based Duty Cycle Assignment) are more energy-efficient than the constant duty cycle method, and that DDCA performs better than TDDCA. DDCA reduces energy dissipation between $21 \%$ and $32 \%$ compared to the constant duty cycle method, while TDDCA reduces energy dissipation between $12 \%$ and $19 \%$ compared to the constant duty cycle method.

Muralidhar Medidi and Yuanyuan Zhou [6] provided a differential duty cycle approach that is designed based on energy consumed by both traffic and idle listening. It assigns different duty cycles for nodes at different distances from the base station to address the energy hole problem, improve network lifetime, and also to maintain network performance. In [7], Francesco Zorzi et. al analyzed the impact of node density on the energy consumption in transmission, reception and idle-listening in a network where nodes follow a duty cycle scheme. They considered the energy performance of the network for different scenarios, where a different number of nodes and different values of the duty cycle are taken into account. In [8], Joseph Polastre et. al proposed B-MAC, a carrier sense media access protocol for wireless sensor networks, that provides a flexible interface to obtain ultra low power operation, effective collision avoidance, and high channel utilization. To achieve low power operation, B-MAC employs an adaptive preamble sampling scheme to reduce duty cycle and minimize idle listening. They compared B-MAC to conventional 802.11- inspired protocols, specifically SMAC. B-MAC's flexibility results in better packet delivery rates, throughput, latency, and energy consumption than S-MAC is also shown. 
Reliable Data Transport in Sensor Networks (RMST) [9] is proposed to provide reliability at the transport layer. This scheme adds reliable data transfer to directed diffusion. RMST is designed for delivering large blocks of data in multiple segments from a source node to a sink node. For example, this is required when time series data has to be transmitted. Reliability in RMST refers to the eventual delivery to all subscribing sinks of any and all fragments related to a unique RMST entity. A unique RMST entity is a data set consisting of one or more fragments from the same source. RMST does not include any real-time guarantees. In RMST, receivers are responsible for detecting whether or not a fragment needs to be re-sent. In the cached mode, the sink node and all intermediate nodes on an enforced path cache segments and check the cache periodically for missing segments. When a node detects missing segments, it generates a NACK message, which travels back to the source along the reinforced path. The first node $A$, having missing segments in its cache, forwards them again towards the sink (and thus towards the requesting node).

\section{PROPOSED MODEL}

\section{Energy Consumed by the Nodes}

The total energy consumed by a node consists of the energy consumed for receiving $\left(E_{\mathrm{rx}}\right)$, transmitting $\left(\mathrm{E}_{\mathrm{tx}}\right)$, sleeping $\left(\mathrm{E}_{\text {sleep }}\right)$, and idle listening $\left(\mathrm{E}_{\mathrm{l}}\right)$.

Total energy consumed is given by

The energy consumed for transmission $\left(E_{t x}\right),[10]$,

$$
\mathrm{E}=\mathrm{E}_{\mathrm{rx}}+\mathrm{E}_{\mathrm{tx}}+\mathrm{E}_{\text {sleep }}+\mathrm{E}_{\mathrm{l}}
$$

$$
\mathrm{E}_{\mathrm{tx}}=\mathrm{E}_{\mathrm{elec}} \times \mathrm{k}+\varepsilon_{\mathrm{amp}} \times \mathrm{k} \times \mathrm{d}^{2}
$$

where, $E_{\text {elec }}$ is the transmitter electronics, $k$ is the Data bit, $\varepsilon_{\mathrm{amp}}$ is transmit amplifier, $\mathrm{d}$ is the distance between nodes.

The energy consumed for receiving $\left(\mathrm{E}_{\mathrm{rx}}\right)$,

$$
\mathrm{E}_{\mathrm{rx}}=\mathrm{E}_{\text {elec }} \times \mathrm{k}
$$

\section{New Energy Efficient Reliable Data Transfer Technique}

In WSNs, the data flows are from both sink-to-sensor nodes and sensor nodes to sink. The traffic pattern is more from sensor nodes to sink in which the sensor nodes forward the sensed data to the sink. In most of the networks hop-byhop mechanism is used to transfer the data. Due to increase in collisions, delay and energy consumption the performance of the network decreases. To reduce the redundancy in forwarded packets, techniques such as Data Aggregation are used in which the data packets are aggregated at a central nodes and the correlated data is transferred from one node to another node. The loss of the single packet would result in a huge amount of data loss. To remove this type of losses the protocol is designed that is energy efficient and improves the packet level reliability.

In this paper, the base idea of monitors presented in [2] is used. By incorporating a duty cycling technique for energy saving the energy efficiency of the network is improved. The energy consumed in the idle listening is saved due to the introduction of duty cycle in the network. The energy consumption in the idle listening is comparable to that of energy consumed for receiving the data packets; the major contribution of energy consumption comes from idle listening. By choosing only a subset of active sensor nodes for data forwarding improves the network lifetime by reducing the energy consumption.

\section{Monitor Configuration for Reliable Data Transfer}

Some initial setup must be followed by all the sensor nodes to make sure that the data is reliably delivered to the base station. The initial setup process is:

1. Identify active and inactive nodes by choosing a subset of nodes.

2. Constructing a data-gathering tree

3. Establishing active data paths with the base station

By carefully choosing the subset of active sensor nodes, the energy consumption of the entire network reduces. This helps in energy savings and the collisions during data forwarding are contained and the network performance is also improved in terms of throughput as the number of nodes is reduced. The nodes that are actively participating in data forwarding the status of that node is set as active and the remaining nodes status is set as inactive. Consider Figure 6 to Figure 8 to better illustrate the initial setup. Figure 6 consists of entire sensor nodes in the network. Node represented by red shade is represents the Base Station. Inactive nodes are represented in dark shade and an active node is in light shade in the Figure7. 


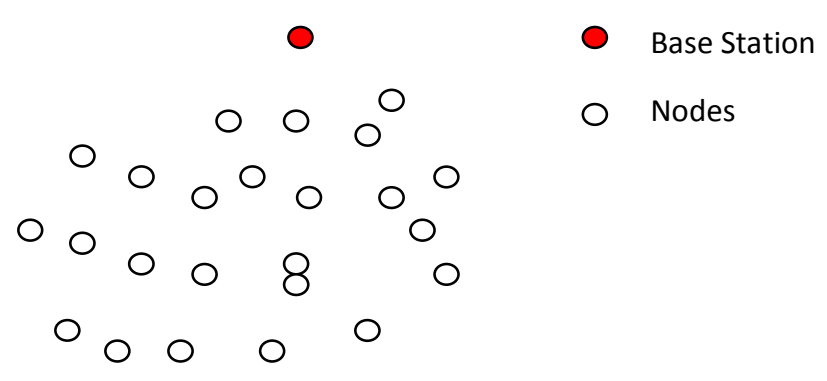

Figure 6 Wireless Sensor Networks

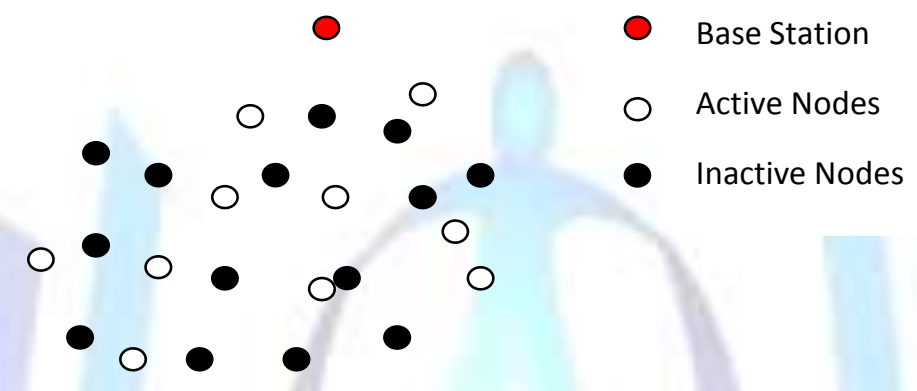

Figure 7 Subset of Nodes

To form the path for all the active nodes, the Base Station initiates and broadcasts a FORWARDER-REQ-MESG and all the nodes that receive this message set their forwarder as Base Station and set their corresponding hop distance from the Base Station. Inactive sets of nodes do not take part in relaying FORWARDER-REQ-MESG down the network.

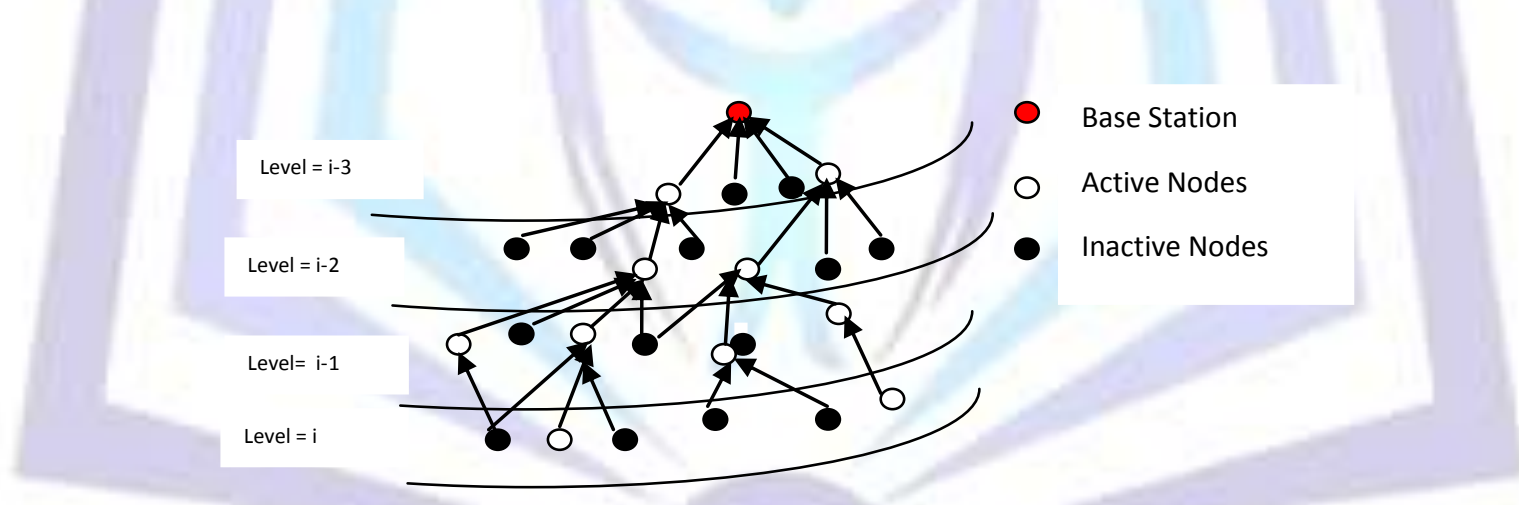

Figure 8 Data Paths

To avoid collisions, within a reasonable time frame a node will randomly choose a time to send the packet. Every node sends these messages only three times, so that it can limit the sent messages from going in a loop. In Figure 9 the final data path is represented. For example, active data paths are $\{V \rightarrow H \rightarrow C \rightarrow B S\}$ and, $\{R \rightarrow M \rightarrow D \rightarrow A \rightarrow B S\}$ and inactive Node forwarder are $\mathrm{G} \rightarrow \mathrm{BS}, \mathrm{I} \rightarrow \mathrm{A}$ and $\mathrm{K} \rightarrow \mathrm{C}$ and so on. 


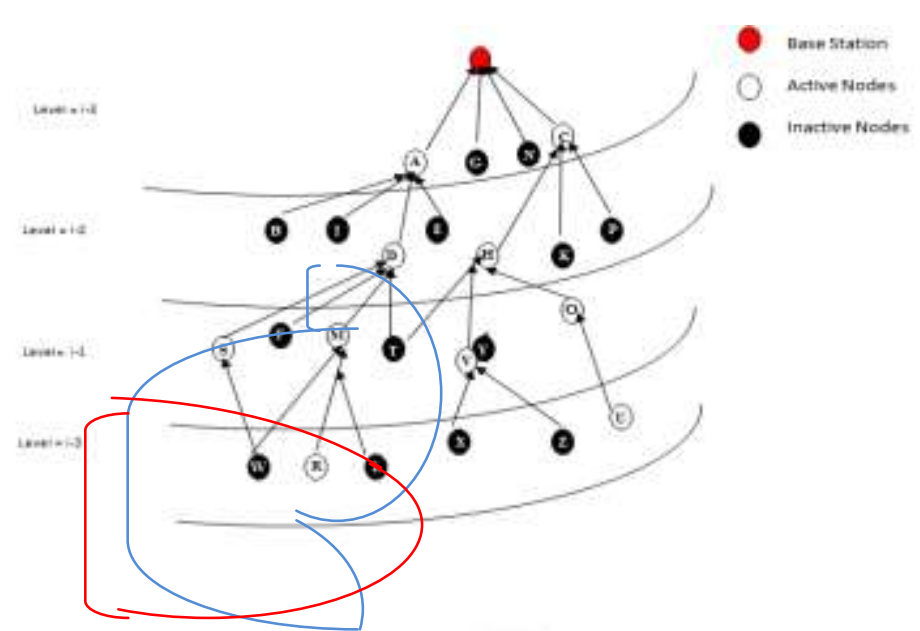

Figure 9 Common Inactive Neighbors

In WSN, sensor nodes mostly use battery power, which makes them energy constrained. If the nodes die due to depletion of their energy, the performance of the network decreases. The nodes in idle listening mode in WSN consume a lot of energy even more than the energy required to receive the data. In WSN, energy efficient techniques must be incorporated, in order to extend the lifetime of the entire network. The energy consumption of the network is reduced by duty-cycle mechanisms, wherein nodes go to sleep and wake up periodically for data transmissions/receptions, are employed.

From Figure 9, consider the nodes $R$ and $M$, in which node $R$ forwards the data to $M$. If duty cycles are introduced, node $R$ and $M$ go to wake-up/sleep cycles periodically. For the active path $R \rightarrow M \rightarrow D \rightarrow A \rightarrow B S$, the wakeup/sleep cycles are shown in Figure 10. The staggered and synchronized duty cycles is assigned in the network. The amount of energy wasted during this unnecessary wake-up can be reduced by employing staggered duty cycles. In staggered duty cycles, all the nodes that are of same hop level wake-up or go to sleep.

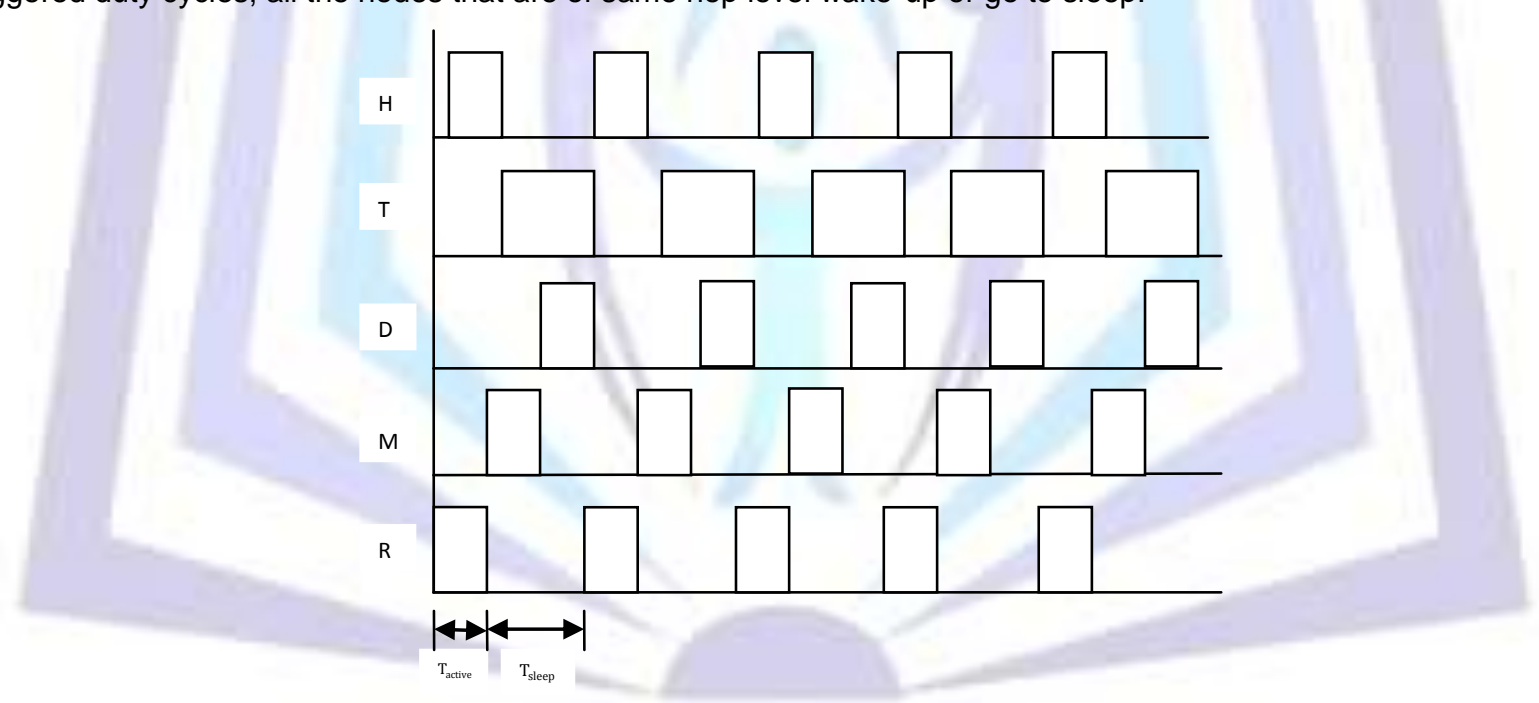

Figure 10 Staggered and Synchronized Duty Cycles

\section{Performance Study}

To evaluate the performance, the protocol is implemented in the MATLAB. In this, the comparison of protocol with the previous monitor-based protocol in [2] in terms of energy consumption, throughput and packet delivery ratio is provided. 


\section{Simulation Setup}

The simulations were run with the simulation parameters as mentioned in Table I. In the scenario there are 100 nodes distributed randomly the terrain area is taken as $100 \mathrm{~m} \times 100 \mathrm{~m}$ as shown in Figure 11 . The initial energy of each node is assumed as 1 Joule. ID is also assigned to each of the nodes.

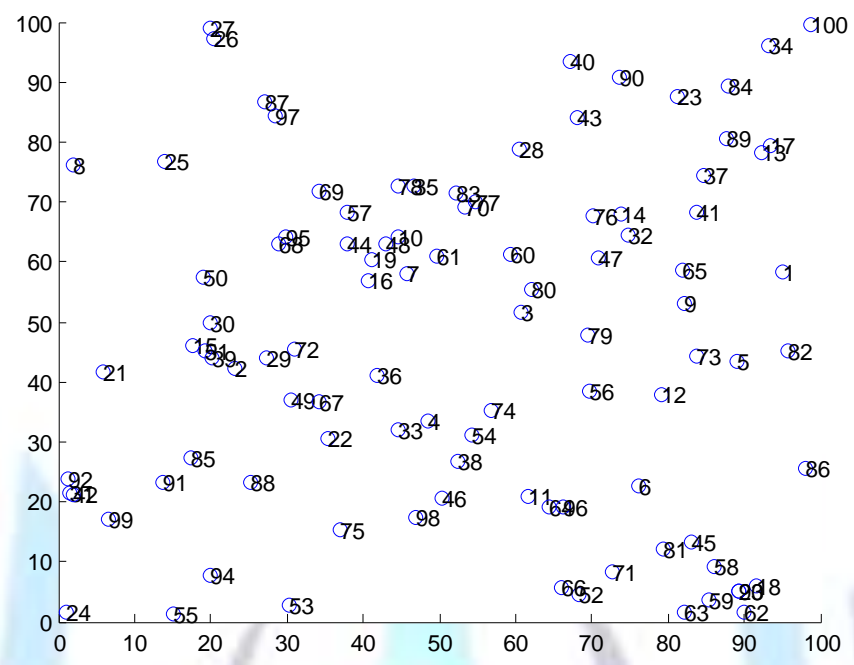

Figure 11100 Nodes Created

Node 100 is considered as the Base Station as shown in Figure 12. The whole network is divided into sub layers.

\begin{tabular}{|c|c|}
\hline Parameter & Value \\
\hline Area & $100 \mathrm{~m} \mathrm{X} \mathrm{100m}$ \\
\hline Total Number of Nodes & 100 nodes \\
\hline Packet interval rate & $0.1(5 \mathrm{~KB} / \mathrm{s})$ to $1(0.5 \mathrm{~KB} / \mathrm{s})$ \\
\hline Transmitter Electronics $\left(\mathrm{E}_{\text {elec }}\right)$ & $50 \mathrm{~nJ}$ \\
\hline Transmit Amplifier $\left(\varepsilon_{\text {amp }}\right)$ & $100 \mathrm{pJ}$ \\
\hline Data Bit $(\mathrm{k})$ & $100 \mathrm{bit}$ \\
\hline Energy for Sleep $\left(\mathrm{E}_{\text {sleep }}\right)$ & 0.000016 \\
\hline Idle Listening Energy $\left(\mathrm{E}_{\mathrm{l}}\right)$ & 0.01236 \\
\hline
\end{tabular}

Table1. Simulation Parameters 


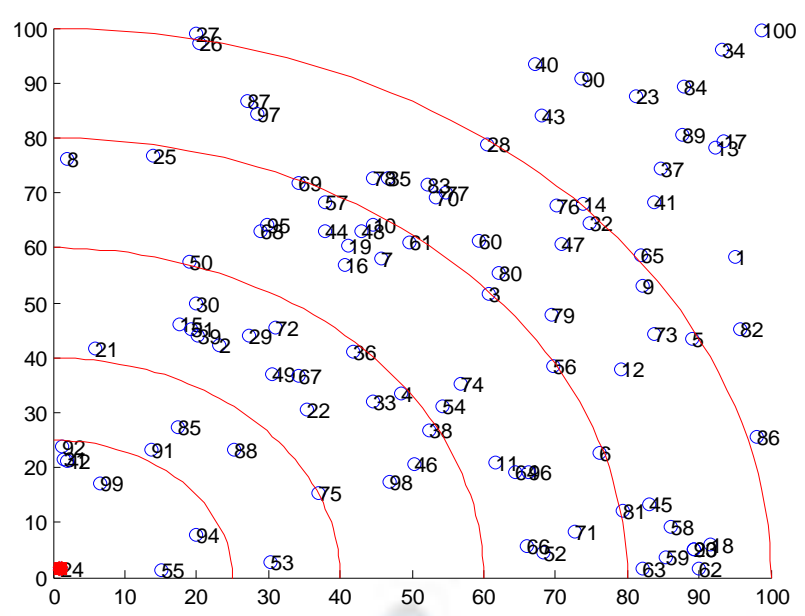

Figure 12 Base Station

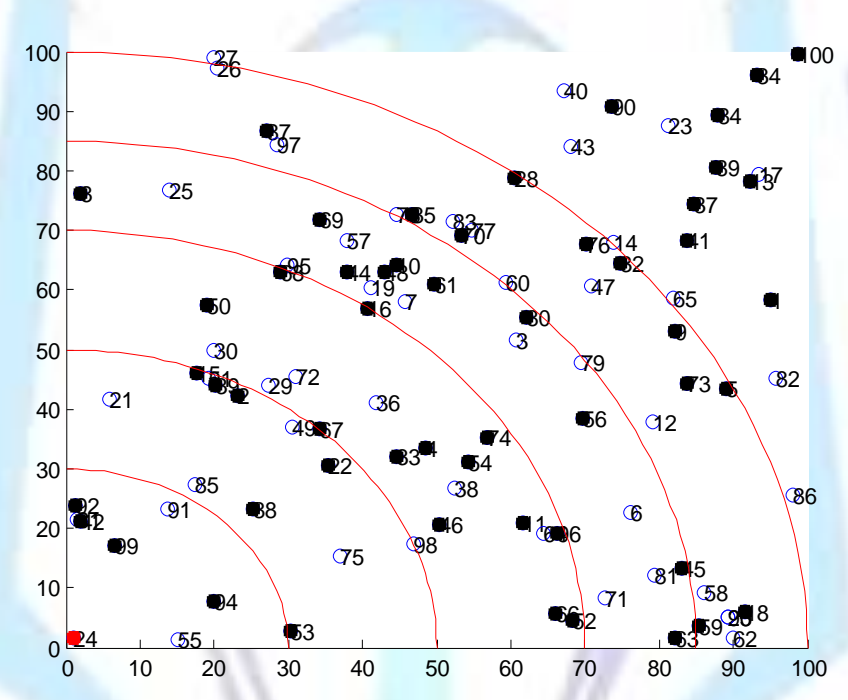

Figure 13 Active and Inactive nodes

All active nodes as shown in Figure 13 take part in Data forwarding and the remaining nodes named as inactive nodes do not take part in data forwarding. Inactive nodes are represented in dark shade and active nodes are in light shade. A subset of 50 nodes was considered to be active nodes and the remaining nodes were considered as inactive nodes as shown in Figure 13. The data path is represented in Figure 14. 


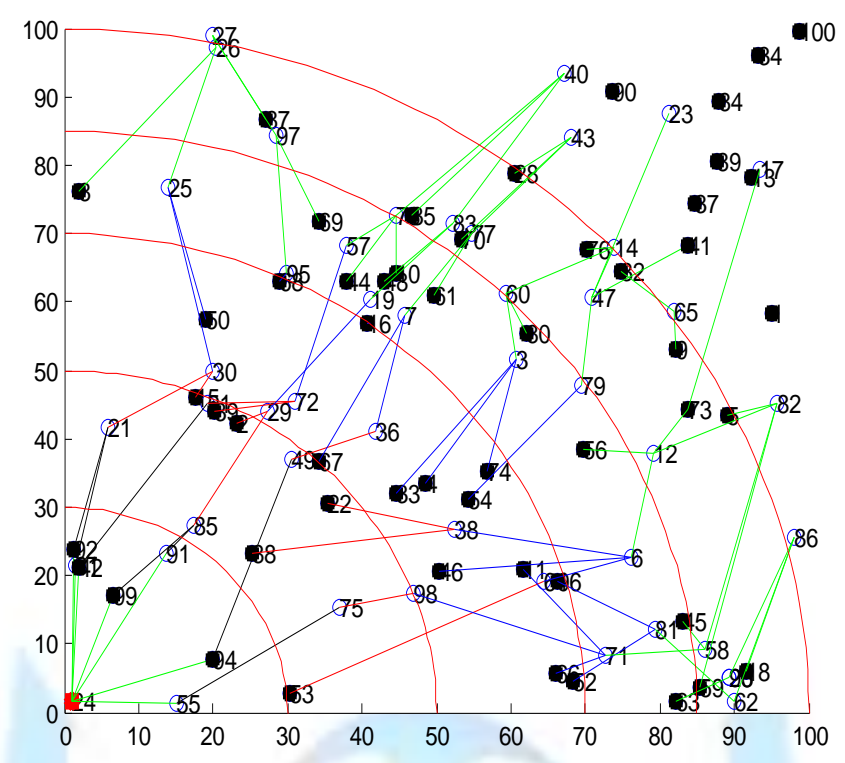

Figure14 Data Paths

To analyze the network 10 active nodes are considered to be sending the data packets at a time.

For the comparison of the new model with the previous model, the simulations were run with the simulation parameters as same as mentioned in Table I. The data path of the previous monitor based approach.

For a meaningful comparison of the new model with [2] in persistent and transient congestion scenarios, metrics such as energy consumption and throughput were considered.

\section{Results}

The New Model is compared with the previous monitor-based approach [2] to evaluate the network performance. Figure 15 and Figure 16 show the comparison of new model in terms of energy consumption and throughput. Figure 15 shows that new model has more energy savings when compared to the previous monitored model. As the data packets size increases the energy consumption increases in the previous monitoring model but in the new model the energy consumption remains almost the same with some minute variations for all the data packets size.

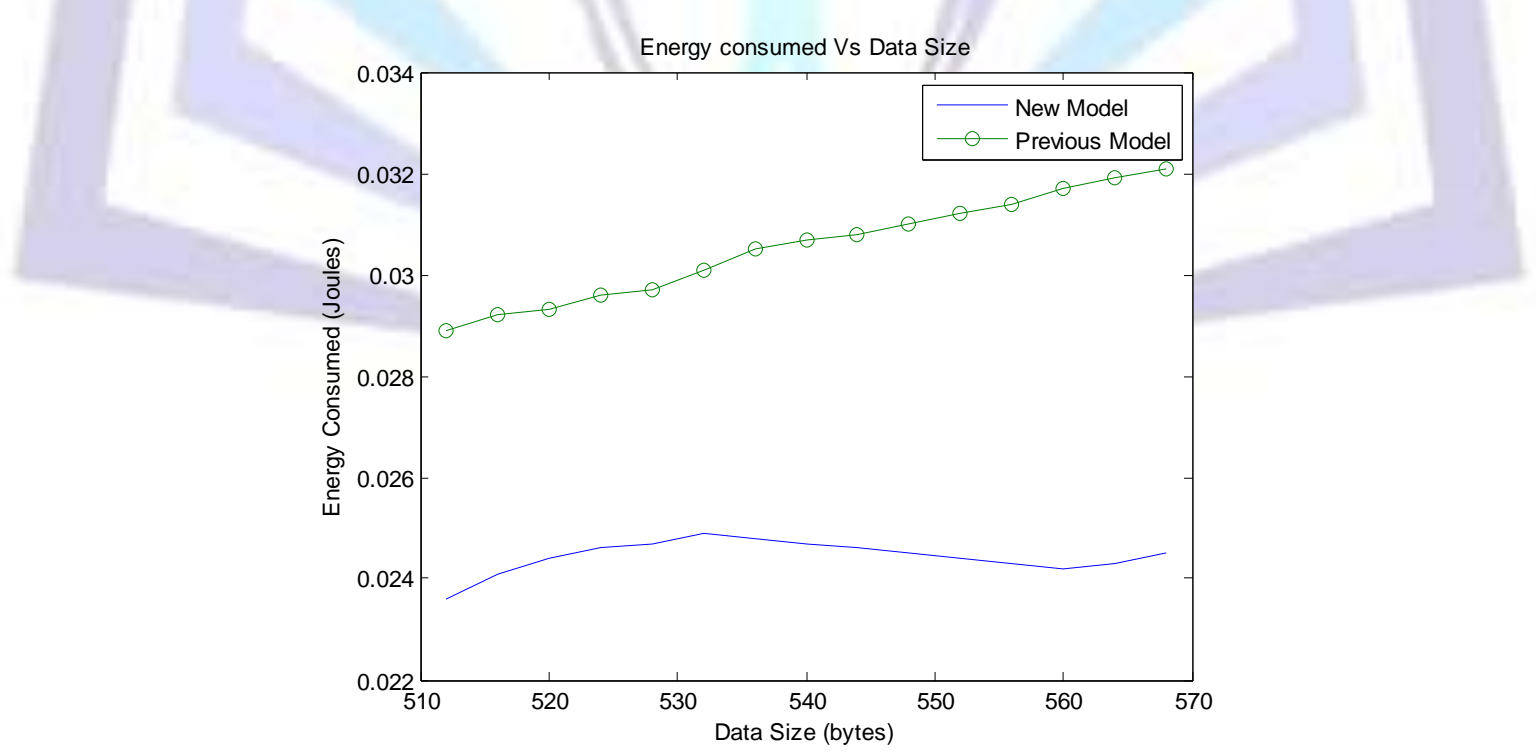

Figure 15 Energy Consumption vs Data Size

Figure 16 shows the improvement in throughput when compared with the previous model. This is because the new model uses the monitors for reliable data delivery. As monitors choose alternate routes for forwarding the data, 
average hop count increases, which in turn increases the packet arrival time and due to this more packets are reliably transferred.

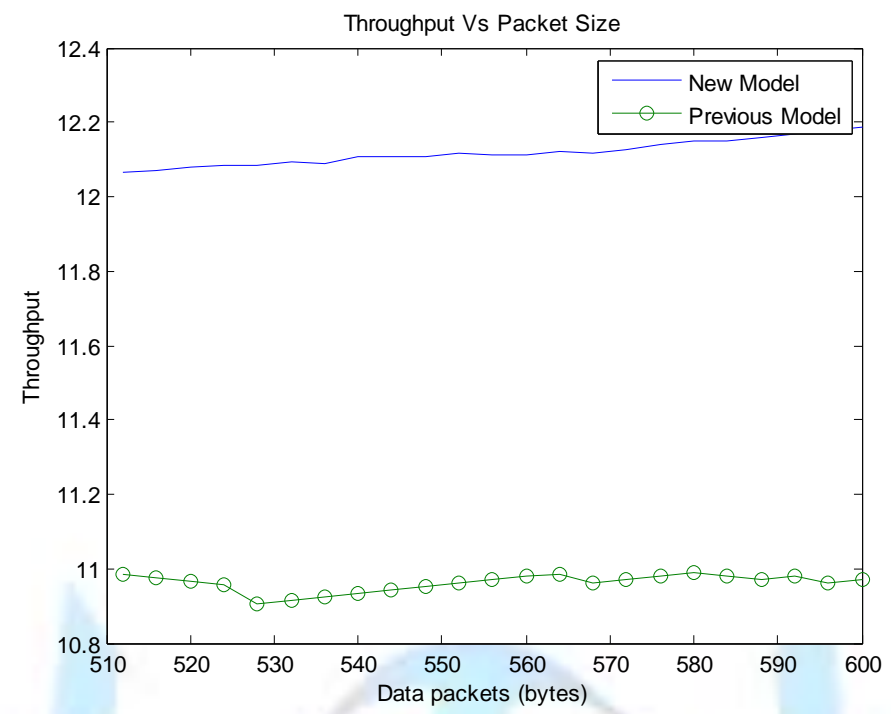

Figure 16 Throughput vs Data Packets Size

Figure 17 shows the plot between packet interval and energy consumption. The new model achieves an increase in energy savings when compared to the previous model.

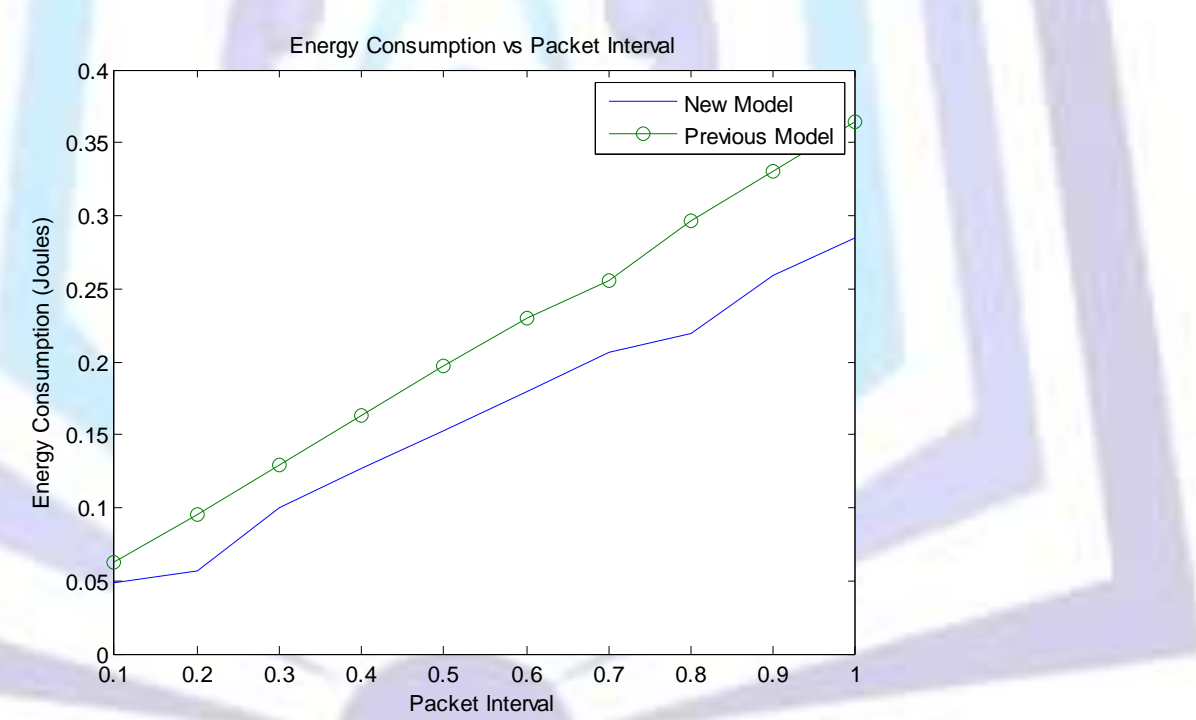

Figure 17 Energy Consumption vs Packet Interval

\section{Effect of duty Cycle}

To evaluate the performance of new model for energy efficiency while maintaining reliable data packets, the new model is evaluated with duty cycle. Figure 18 shows that with the duty cycling mechanism, the energy consumption of the network decreases drastically. 


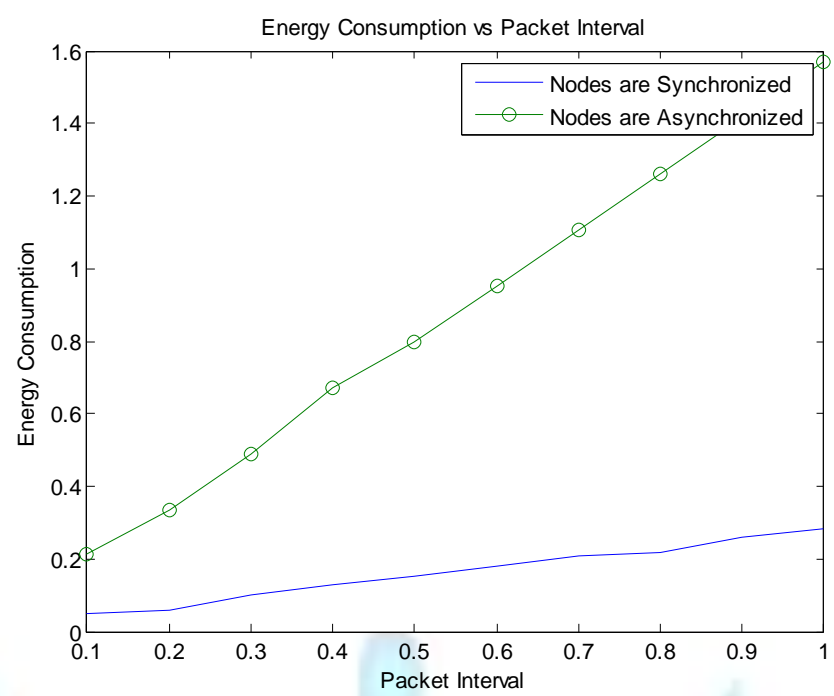

Figure 18 Energy consumption vs Packet Interval (Duty Cycle)

Figure 19 shows the plot between packet delivery ratio vs Packet Interval. Packet Delivery ratio is defined as the total number of packets successfully delivered to the base station to the total number of packets generated is measured. This packet-delivery ratio gives the measure of reliable packet transfer. Figure 19 shows that the packet delivery ratio is more in the network which consist of monitors. This is because of the reduction in collisions due to the introduction of the monitors.

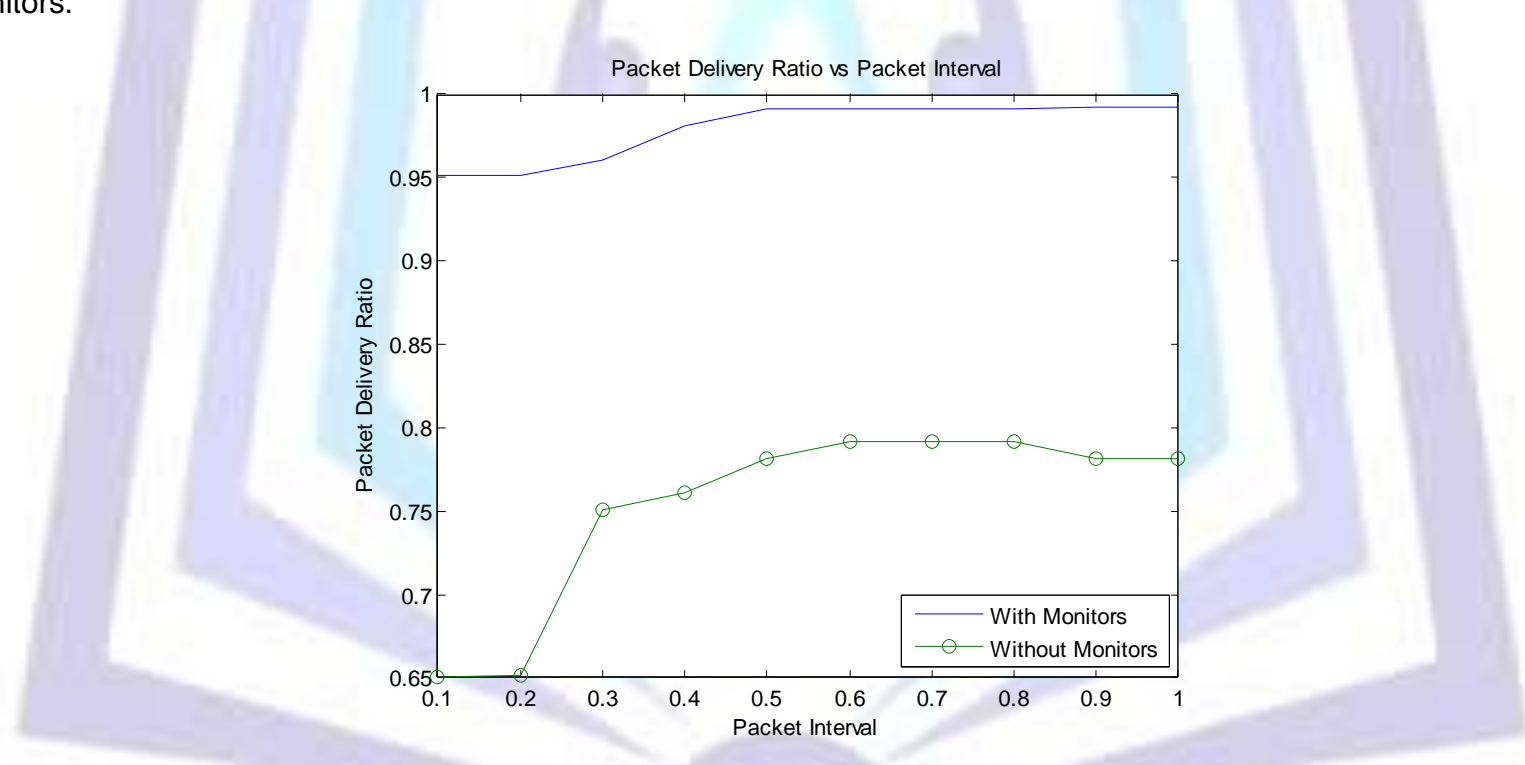

Figure 19 Packet Delivery Ratio vs Packet Interval

\section{Conclusions}

Wireless Sensor Networks are mainly deployed for monitoring purposes in various fields. In many applications, data aggregation techniques are used to reduce the redundancy in forwarded packets. In these techniques, at intermediate nodes the packets are aggregated and the correlated data is forwarded from one node to another. Hence, there arises a need for a packet level reliable data transport protocol. Here, a reliable data transfer protocol by configuring inactive nodes as monitors is developed to enhance the packet-level reliability and reduce energy consumption. In this protocol, the synchronized duty cycle is also introduced by which the energy consumption of the protocol decreases drastically. Results show that this technique improves energy efficiency, throughput and the packet delivery ratio even under congested scenarios.

In the future, the monitor-based approach to select the monitors based on location and the coverage density would be attempted. To further improve energy efficiency a very low duty cycle can be used. 


\section{REFERENCES}

[1] Ajay Jangra, Swati, Richa, Priyanka, "Wireless Sensor Network (WSN) Architectural Design issues and Challenges," International Journal on Computer Science and Engineering, Vol. 02, Issue. 09, pp. 30893094, 2010.

[2] J. Wang and S. Medidi, "Topology Control for Reliable Sensor-to-Sink Data Transport in Sensor Networks," in Proceedings of the IEEE International Conference on Communications (ICC), pp. 32153219, 2008.

[3] Honghai Zhang and Jennifer C. Hou, "Maximizing $\alpha$-Lifetime for Wireless Sensor Networks," $\underline{\text { IJSNet }}$ 1(1/2), Vol.1, Iss 1, pp. 64-71, January 2006.

[4] MS Pawar, JA Manore, MM Kuber, "Life Time Prediction of Battery Operated Node for Energy Efficient WSN Applications," IJCST Vol. 2, Issue 4, Oct. - Dec. 2011.

[5] Yuqun Zhang, Chen-Hsiang Feng, llker Demirkol, Wendi Rabiner Heinzelman, "Energy-Efficient Duty Cycle Assignment for Receiver-Based Convergecast in Wireless Sensor Networks," publication in the IEEE Globecom proceedings, pp. 1-5, 2010.

[6] Muralidhar Medidi, Yuanyuan Zhou, "Extending Lifetime with Differential Duty Cycles in Wireless Sensor Networks," IEEE Communications Society subject publication, pp.1033-1037, May 7, 2009.

[7] Francesco Zorzi, Milica Stojanovic and Michele Zorzi, "On the Effects of Node Density and Duty Cycle on Energy Efficiency in Underwater Networks," Conference Europe, pp.1-6, 24-27 May, 2010.

[8] Joseph Polastre, Jason Hill, David Culler, "Versatile Low Power Media Access for wireless," sensor Networks, The Second ACM Conference on Embedded Networked Sensor Systems, pp. 95-107, Nov. 2004.

[9] F.Stann and J.Heidemann. Rmst, "Reliable data transport in sensor networks," Anchorage, Alaska, USA., SNPA (Sensor Net Protocols and Applications), pp.1-11, May 11, 2003.

[10] Sung-Min Jung, Young-Ju Han, Tai-Myoung Chung, "The Concentric Clustering Scheme for Efficient Energy Consumption in the Pegasis,” pp. 260-265, February 12-14, 2007.

\section{Author' biography with Photo}

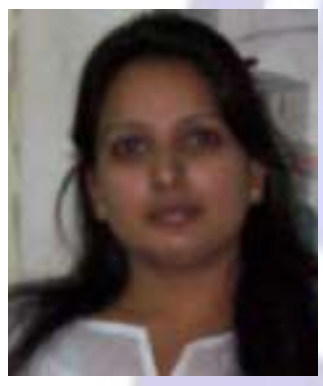

Jyoti Saraswat was born in India on November 26, 1987. She received her B.Tech degree in Electronics and Communication Engineering from Modi Institute of Engineering and Technology, Rajasthan University, India in 2010 and currently is a M. Tech (Signal Processing) student in Mody Institute of Technology and Science (Deemed University), Rajasthan, India.

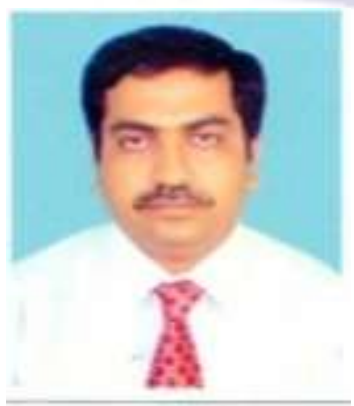

Dr. Partha Pratim Bhattacharya was born in India on January 3, 1971. He has 16 years of experience in teaching and research. He served many reputed educational Institutes in India in various positions. At present he is working as Professor in Department of Electronics and Communication Engineering in the Faculty of Engineering and Technology, Mody Institute of Technology and Science (Deemed University), Rajasthan, India. He worked on Microwave devices and systems and mobile cellular communication systems. He has published more than 95 papers in refereed journals and conferences. His present research interest includes mobile cellular communication, wireless sensor network and cognitive radio.

Dr. Bhattacharya is a member of The Institution of Electronics and Telecommunication Engineers, India and The Institution of Engineers, India. He is the recipient of Young Scientist Award from International Union of Radio Science in 2005. He is working as the chief editor, editorial board member and reviewer in many reputed journals. 